\title{
Convalidando Instrumento de Avaliação da Qualidade da Modalidade a Distância por meio da Teoria Sistêmica e das Disciplinas Semipresenciais
}

\author{
Júlio C. G. Bertolin - ppgEdu/UPF - julio@upf.br \\ Ana Carolina Bertoletti De Marchi - ppgCA/ppgEH/UPF - carolina@upf.br
}

\begin{abstract}
Resumo: No interstício das modalidades a distância e presencial está a semipresencialidade que geralmente desenvolve momentos a distância num contexto preponderante de aulas presenciais, fazendo com que a avaliação da qualidade da $\mathrm{EaD}$ encontre nas disciplinas semipresenciais um contexto privilegiado para o desenvolvimento de comparações com a modalidade presencial. Nesse contexto, este artigo valida um instrumento de avaliação de disciplinas semipresenciais fundamentado em indicadores sistêmicos, com vistas a contribuir com o desenvolvimento da avaliação e dos indicadores de qualidade da EaD. O aspecto metodológico utilizado foi baseado na Teoria Geral dos Sistemas através do cotejamento entre os indicadores de resultado e os aspectos de entrada e processo das disciplinas.
\end{abstract}

Palavras-chave: Educação a distância. Semipresencialidade. Avaliação da qualidade da EaD. Indicadores de qualidade da EaD.

\section{Validating Instrument for Quality Assessment of Open and Distance Learning by Systemic Theory and Hybrid Disciplines}

\begin{abstract}
In the interstitium of the Open and Distance Learning and classroom teaching are hybrid disciplines which usually develops times the distance in the context of dominant classroom, making the evaluation of the quality of distance education in the hybrid disciplines find an ideal context for the development of comparisons with the classroom teaching. In this context, this paper validates an assessment tool based on hybrid disciplines systemic indicators, in order to contribute to the development and evaluation of the quality of Open and Distance Learning. The methodological aspect was based on general systems theory through the comparison between outcome indicators and aspects of input and process disciplines.
\end{abstract}

Keywords: Open and Distance Learning. Hybrid disciplines. Quality assessment of ODL. Quality indicators in ODL.

\section{Introdução}

A avaliação é um tema complexo que possui importância significativa nas abordagens e projetos de qualidade de instituições e cursos de educação superior. Da mesma forma que existem diferentes visões de educação superior e de qualidade em 
educação, também existem diferentes concepções e formas de operacionalizar a avaliação da educação. Nas últimas duas décadas, debates acerca da avaliação da educação superior adquiriram grande proeminência, envolvendo profissionais e pesquisadores de diferentes áreas do conhecimento. Entretanto, no âmbito da modalidade de $\mathrm{EaD}$ os estudos e trabalhos sobre avaliação ainda são relativamente incipientes. Quando abordada no âmbito da modalidade a distância, a avaliação da qualidade da educação adquire maior complexidade e importância visto que as diferenças de espaço e tempo entre professor e aluno certamente impactam o processo de aprendizagem.

As abordagens implementadas para a avaliação da EaD vão desde a comparação dos resultados obtidos pela modalidade a distância com os obtidos pela aprendizagem tradicional e presencial; passando por dispositivos para armazenar e analisar a duração e a frequência de login ou páginas acessadas; até o uso de indicadores para avaliar a plataforma ou ambiente virtual de aprendizagem (Valcheva e Todorova, 2005). Assim, de maneira geral, as pesquisas sobre qualidade da $\mathrm{EaD}$ estão centradas, fundamentalmente, na comparação com os "resultados" da educação presencial ou na avaliação do "processo" dos ambientes de aprendizagem. São raras as avaliações da qualidade da $\mathrm{EaD}$ que abordem, de forma concomitante, todos os aspectos sistêmicos do desenvolvimento da modalidade, ou seja, que utilizem indicadores de entrada, processo e resultado simultaneamente. Bertolin e De Marchi (2009) elaboraram uma proposta do uso destes indicadores para avaliar disciplinas semipresenciais em cursos de graduação.

No interstício das modalidades a distância e presencial está a semipresencialidade que geralmente desenvolve momentos a distância num contexto preponderante de aulas presenciais. Desde 2004, através da Portaria 4.059, o Ministério da Educação do Brasil autoriza que até $20 \%$ do total da carga horária de um curso de graduação presencial reconhecido seja desenvolvida na modalidade a distância. Dentro do escopo de uma disciplina, a legislação permite que a modalidade a distância seja desenvolvida integral ou parcialmente, resguardadas as avaliações que devem ser presenciais. Assim, uma disciplina semipresencial, não obstante a predominância de aulas tradicionais, possui níveis significativos de flexibilização do processo de aprendizagem em relação ao tempo e ao espaço, o que consubstancia uma condição privilegiada para investigação da qualidade da EaD visto que um mesmo grupo de alunos vivencia, numa mesma disciplina e com um mesmo professor, momentos intercalados entre a modalidade a distância e o ensino presencial. Por conseguinte, a avaliação da qualidade da EaD encontra na semipresencialidade um contexto especial para o desenvolvimento de comparações com a modalidade presencial.

Nesse contexto, com vistas a contribuir com o desenvolvimento da avaliação e dos indicadores de qualidade da EaD, este artigo valida um instrumento de avaliação de disciplinas semipresenciais fundamentado em indicadores sistêmicos. O aspecto metodológico utilizado foi baseado na Teoria Sistêmica através do cotejamento entre os indicadores em diferentes disciplinas. Os resultados evidenciaram a coerência e a validade do instrumento na medida em que os níveis das entradas e dos processos coadunaram com os resultados obtidos.

\section{Instrumento e indicadores de avaliação}

Muitos dos instrumentos elaborados para as avaliações da qualidade em educação não possuem um teoria científica como base de concepção. Geralmente, questionários e 
indicadores são definidos a partir do senso comum, percepções subjetivas e experiências empíricas. Um instrumento de avaliação deve ser elaborado de acordo com uma concepção de avaliação e de qualidade em educação e, por conseguinte, orientado por um método que coadune com tais visões de avaliação e qualidade.

Importantes organismos internacionais como a UNESCO e a OCDE têm interpretado uma concepção de qualidade em educação por meio da representação de sistemas de indicadores ou indicadores sistêmicos. Segundo Estrada (1999), a avaliação da qualidade em educação se expressa como um juízo de valor sobre um conjunto de atributos acerca das "entradas”, "processo" e "resultados", ou das relações entre eles. O Laboratorio Latinoamericano de Evaluación de la Calidad de la Educación da UNESCO também se reporta a indicadores sistêmicos ao afirmar que

o nível de qualidade da educação consiste basicamente na definição de um conjunto de variáveis que proporcione, em forma sistemática, um quadro confiável e válido acerca do estado dos sistemas de educação e que pode ser utilizado para colaborar na orientação e ações de melhoria (UNESCO, 1997, p. 7).

De acordo com García (2000), os sistemas de indicadores procuram superar a obtenção de uma simples soma de dados ao agrupar indicadores simples ou compostos em função de fatores e aspectos que lhes deem sentido e uma visão significativa do estado dos sistemas de educação. As experiências de avaliação vinculadas à avaliação com sistemas de indicadores consideram que a qualidade em educação é um conceito múltiplo que não pode ser avaliado por apenas um aspecto isolado e deve envolver todos os elementos fundamentais do sistema ou processo. Assim, pode-se dizer que é possível avaliar a qualidade em educação por meio de juízo de valor baseado na análise de um conjunto de atributos, aspectos ou indicadores acerca das entradas, processo e resultados educativos, ou das relações entre eles.

Geralmente os indicadores de entrada referem-se aos recursos humanos e tecnológicos que se destinam à educação. Aspectos relativos aos investimentos em tecnologias e a quantidade e formação dos docentes também podem estar incluídos entre os indicadores de entrada e/ou recursos. Os indicadores de processo referem-se ao contexto pedagógico e organizacional, ou seja, dizem respeito às características primárias, relativas à participação direta do processo de educação, e secundárias, relativas ao apoio à organização das características primárias. Aspectos relativos ao número de horas de ensino e dedicação dos docentes podem compor a estrutura dos indicadores de processo. Os indicadores de resultados referem-se às características relativas aos propósitos intermediários e últimos da educação; nível de êxito dos alunos em exames e aprendizagem podem estar entre os indicadores de resultados (Estrada, 1999), (European Commission, 2001), (OECD , 2002) e (Navarra, 2004).

Não obstante a existência e a possibilidade de outras formas de organização, os sistemas de indicadores estruturados em entradas, processo e resultados tornaram-se um dos métodos mais empregados para avaliar a qualidade em educação superior, principalmente em nível de sistema de educação. Contudo, tal estrutura de indicadores pode ser empregada também para avaliação de uma instituição, um curso ou mesmo uma disciplina visto que tais níveis de análise podem ser entendidos como sistemas ou subsistemas. Um sistema é um conjunto de elementos interdependentes e interagentes; um grupo de unidades combinadas que formam um todo organizado e cujo resultado é maior do que o resultado que as unidades poderiam ter se funcionassem de forma 
independente. Assim, da mesma forma que as instituições universidades e faculdades podem ser consideradas elementos do "sistema de educação", cursos podem ser considerados elementos do "sistema instituição", disciplinas elementos do "sistema curso" e, por conseguinte, os aspectos constitutivos de uma disciplina, tais como, professor, conteúdo, recursos pedagógicos etc., podem ser analisados como elementos do "sistema disciplina".

Portanto, em face dos objetivos deste artigo, pressupõe-se que a avaliação da qualidade de disciplinas semipresenciais está diretamente relacionada ao desempenho de um conjunto de indicadores de entradas, de processo e de resultados. Ao se realizar uma avaliação da qualidade de uma disciplina também com indicadores de entrada e processo, além dos resultados, a verificação e o "olhar" não se limitam ao objetivo a ser atingido como, por exemplo, a aprendizagem. A abordagem mais ampla, considerando aspectos de entrada e processo, que têm importância fundamental na aprendizagem, permite identificar melhor as causas dos resultados obtidos ou não e, por conseguinte, possibilita a emissão de um juízo de valor mais confiável sobre a qualidade do sistema avaliado ou disciplina em avaliação.

Assim, com o objetivo de contribuir com o desenvolvimento da avaliação da qualidade EaD adotou-se o instrumento proposto por Bertolin e De Marchi (2011) que contempla aspectos sistêmicos de entradas, processo e resultados de uma disciplina semipresencial (Tabela 1). O instrumento, direcionado aos alunos, conta com 14 questões que podem receber conceitos ordinários de ótimo, bom, regular, ruim ou péssimo.

\section{Tabela 1. Instrumento para disciplinas semipresenciais}

\begin{tabular}{l|l}
\hline \multirow{4}{*}{ Entrada } & $\begin{array}{l}\text { 1. A infraestrutura de EaD (ambiente de aprendizagem, recursos de } \\
\text { informática etc.) disponibilizada pela instituição para a disciplina pode } \\
\text { ser avaliada como? }\end{array}$ \\
\cline { 2 - 3 } $\begin{array}{l}\text { 2. As habilidades e competências do professor para o desenvolvimento } \\
\text { da disciplina podem ser avaliadas como? }\end{array}$ & $\begin{array}{l}\text { 3. As suas habilidades de uso de computador antes do início da } \\
\text { disciplina podem ser avaliadas como? }\end{array}$ \\
\cline { 2 - 3 } & $\begin{array}{l}\text { 4. O planejamento (plano de ensino, carga horária a distância, material } \\
\text { didático, bibliografia, mídias etc.) realizado pelo professor para a } \\
\text { disciplina pode ser avaliado como? }\end{array}$ \\
\hline \multirow{5}{*}{ Processo } & $\begin{array}{l}\text { 5. A interação desenvolvida entre professor-aluno durante a disciplina } \\
\text { pode ser avaliada como? }\end{array}$ \\
\cline { 2 - 3 } & $\begin{array}{l}\text { 6. O acompanhamento prestado pelo professor durante o } \\
\text { desenvolvimento da disciplina pode ser avaliado como? }\end{array}$ \\
\cline { 2 - 3 } & $\begin{array}{l}\text { 7. O suporte técnico prestado pela UPF Virtual durante o } \\
\text { desenvolvimento da disciplina pode ser avaliado como? }\end{array}$ \\
\cline { 2 - 3 } $\begin{array}{l}\text { 8. A articulação e a adequação entre os momentos presenciais e a } \\
\text { distância (conteúdos e carga horária) da disciplina podem ser avaliadas }\end{array}$ \\
\hline
\end{tabular}




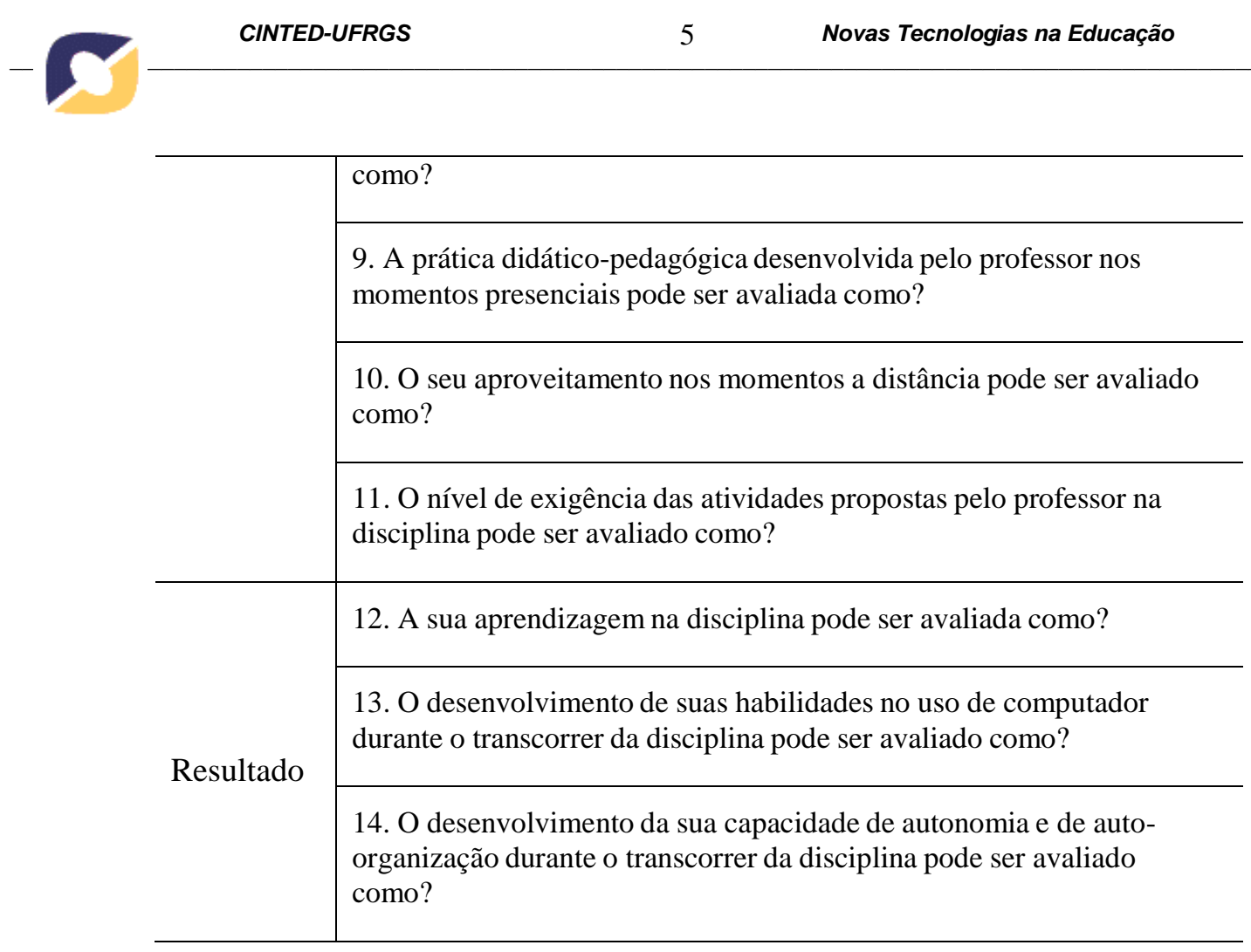

Fonte: Bertolin e De Marchi, 2011.

\section{Método e aplicação do instrumento}

A validação dos indicadores e aspectos sistêmicos de avaliação da qualidade de disciplinas semipresenciais desenvolveu-se por meio da aplicação do instrumento adotado, no primeiro semestre do ano de 2011, aos alunos de diversas disciplinas semipresenciais de uma universidade comunitária, pública não estatal e sem fins lucrativos do Sul do Brasil que possui aproximadamente 20 mil alunos.

A validação do instrumento deste estudo considera os princípios da Teoria Sistêmica, base para sua definição. Segundo tal teoria, um sistema é um conjunto de elementos interdependentes e interagentes; um grupo de unidades combinadas que formam um todo organizado e cujo resultado é maior do que o resultado que as unidades poderiam ter se funcionassem de forma independente (MIZIKACI, 2006). A teoria geral dos sistemas baseia-se na suposição de que existem regras universais de organização que valem para todos os sistemas. O princípio básico da teoria é que o todo é maior que a soma de suas partes, que o todo determina a natureza das partes e que as partes são dinamicamente inter-relacionadas e não podem ser entendidas isoladamente.

Considera-se que os sistemas têm quatro características principais: (i) os sistemas são orientados à meta, (ii) os sistemas têm entradas a partir do ambiente, (iii) os sistemas têm saídas para atingir suas metas e (iv) há feedback enviado pelo ambiente em função das saídas.

Portanto, um sistema não pode ser conhecido pela simples soma de seus elementos, bem como a análise formal de segmentos artificialmente isolados não permite que se compreenda seu funcionamento global. Tais conceitos e características são gerais e devem se aplicar aos mais diversos tipos de sistemas, tais como sistemas sociais, técnicos, organizacionais e, consequentemente, também em sistemas de ensino e educação.

Assim sendo, o instrumento adotado para avaliação de disciplinas semipresenciais será validado por meio do cotejamento entre os indicadores de entrada, processo e 
resultado, ou seja, comparando a coerência do nível de qualidade das entradas e do processo com o nível de qualidade dos resultados. Por exemplo, se os aspectos de entrada e de processo são insatisfatórios, consequentemente os resultados também devem ser. Por outro lado, os resultados devem ser satisfatórios se os níveis de qualidade nos indicadores de entrada e processo forem satisfatórios.

Diante do contexto acima, com objetivo de validar o instrumento e os indicadores propostos, foram tabuladas as respostas de 212 alunos de diversas disciplinas desenvolvidas de forma semipresencial em diferentes cursos da instituição. De maneira geral, as disciplinas tiveram aproximadamente $20 \%$ de sua carga horária desenvolvida a distância e o restante de forma presencial.

Em um primeiro momento foi calculada a média geral de cada disciplina considerando as 14 questões do instrumento de avaliação. Este cálculo teve como objetivo possibilitar o agrupamento das melhores e piores disciplinas com vistas a buscar dados distintos que pudessem comprovar a validação do instrumento. A partir da média geral, as disciplinas foram agrupadas como segue: (i) melhor desempenho: dez disciplinas com maior média; (ii) desempenho intermediário: dez disciplinas em posição mais intermediaria entre as melhores e piores médias; e (iii) pior desempenho: dez disciplinas com pior média.

O cotejamento entre os indicadores/aspectos de "entrada" e "processo" com os indicadores/aspectos de "resultados" foi realizado tendo por base as médias obtidas por cada conjunto de indicadores, agrupadas pelos três grupos de disciplinas acima citados. Assim, foi possível comparar a coerência entre as médias obtidas entre os diferentes tipos de indicadores (entradas, processo e resultados), nos três grupos de disciplinas com diferentes desempenhos (melhor desempenho, desempenho intermediário e pior desempenho) o que, por conseguinte, possibilitou a validade do próprio instrumento.

\section{Medições e resultados obtidos}

Com vistas a gerar médias de desempenho das disciplinas nos diferentes tipos de indicadores considerou-se que uma resposta "ótimo" a uma questão do instrumento equivaleria ao valor 5, uma resposta "bom" valor 4, "regular" valor 3, "ruim" valor 2 e "péssimo" valor 1. A seguir, procedeu-se a média obtida por cada disciplina em cada um dos três tipos de indicadores (entrada, processo ou resultado). Por fim, foi realizado o cálculo da média obtida por cada um dos três conjuntos de disciplinas consideradas (as dez de melhor desempenho, as dez com desempenho intermediário e as dez com pior desempenho) para cada tipo de indicador (entrada, processo ou resultado).

As médias obtidas pelas disciplinas que obtiveram os dez melhores desempenhos nos três tipos diferentes de indicadores/aspectos foram muito semelhantes (Figura 1). Após arredondamentos, as entradas ficaram com média 4,5, os aspectos de processo com média 4,5 e os indicadores de resultados com média 4,5, ou seja, a qualidade dos resultados nessas disciplinas foram coerentes com o nível avaliado para as entradas e o processo. 


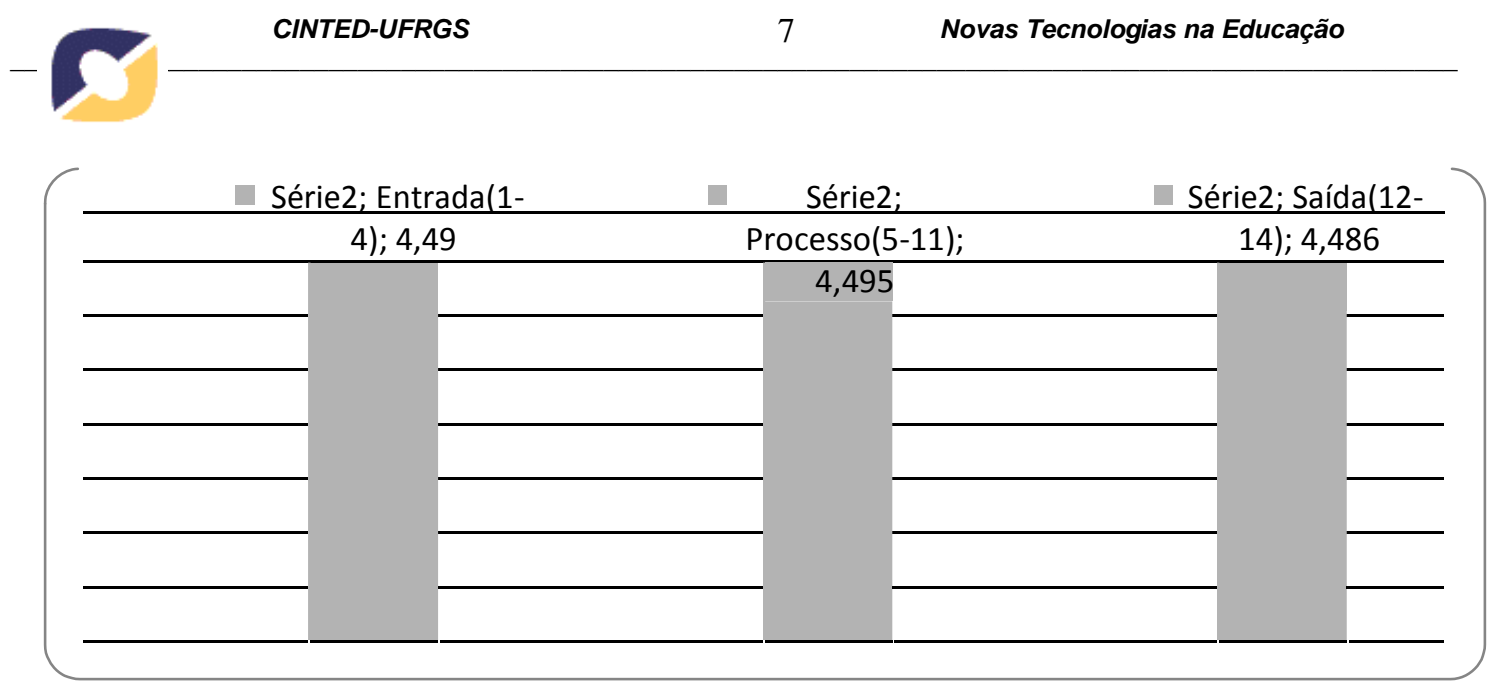

Figura 1. Médias das dez disciplinas com melhor desempenho

As médias obtidas pelas disciplinas que tiveram um desempenho intermediário nos três tipos diferentes de indicadores/aspectos também foram semelhantes (Figura 2). Com arredondamentos, as entradas ficaram com média 4,1, os aspectos de processo com média 4,1 e os indicadores de resultados com média 4,0. Em síntese, o nível da qualidade das entradas e do processo estão coerentes com o nível dos resultados obtidos.

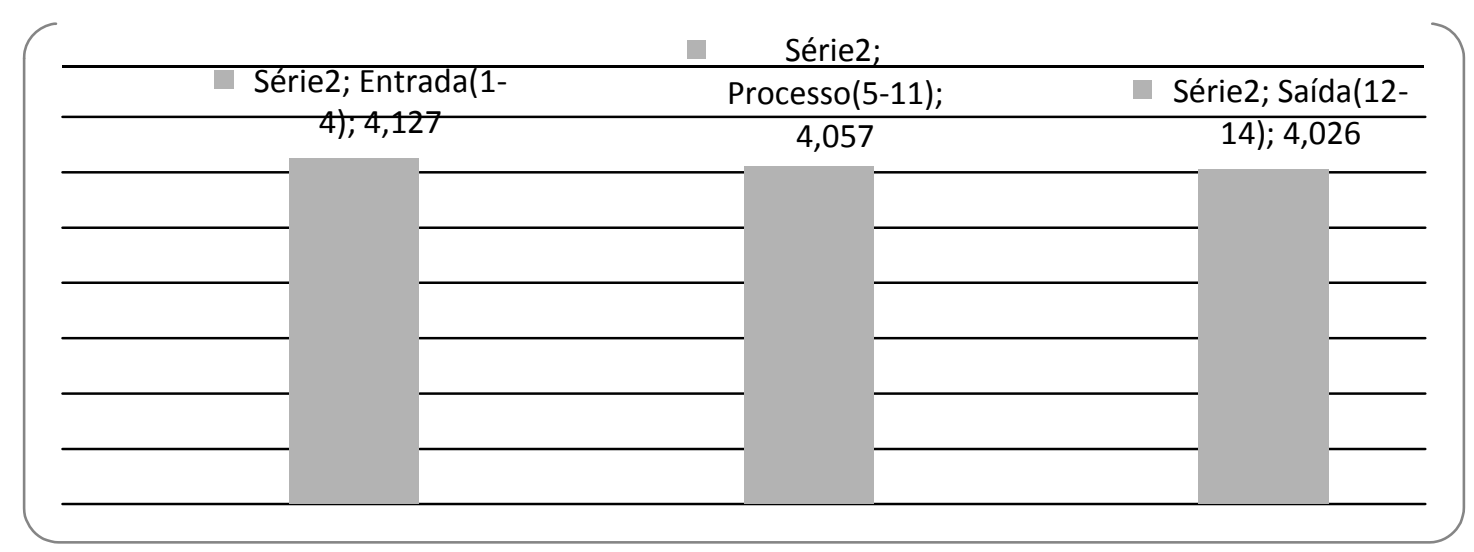

Figura 2. Média das dez disciplinas com desempenho intermediário

Da mesma forma, as médias obtidas pelas disciplinas com pior desempenho nos três tipos diferentes de indicadores/aspectos foram semelhantes (Figura 3). Após arredondamentos, as entradas ficaram com média 3,5, os aspectos de processo com média 3,2 e os indicadores de resultados com média 3,4. As médias dos três tipos de indicadores/aspectos ficaram muito próximas da média geral da disciplina de 3,4, ou seja, também entre as disciplinas de pior desempenho os resultados foram coerentes com os níveis das entradas e do processo. 


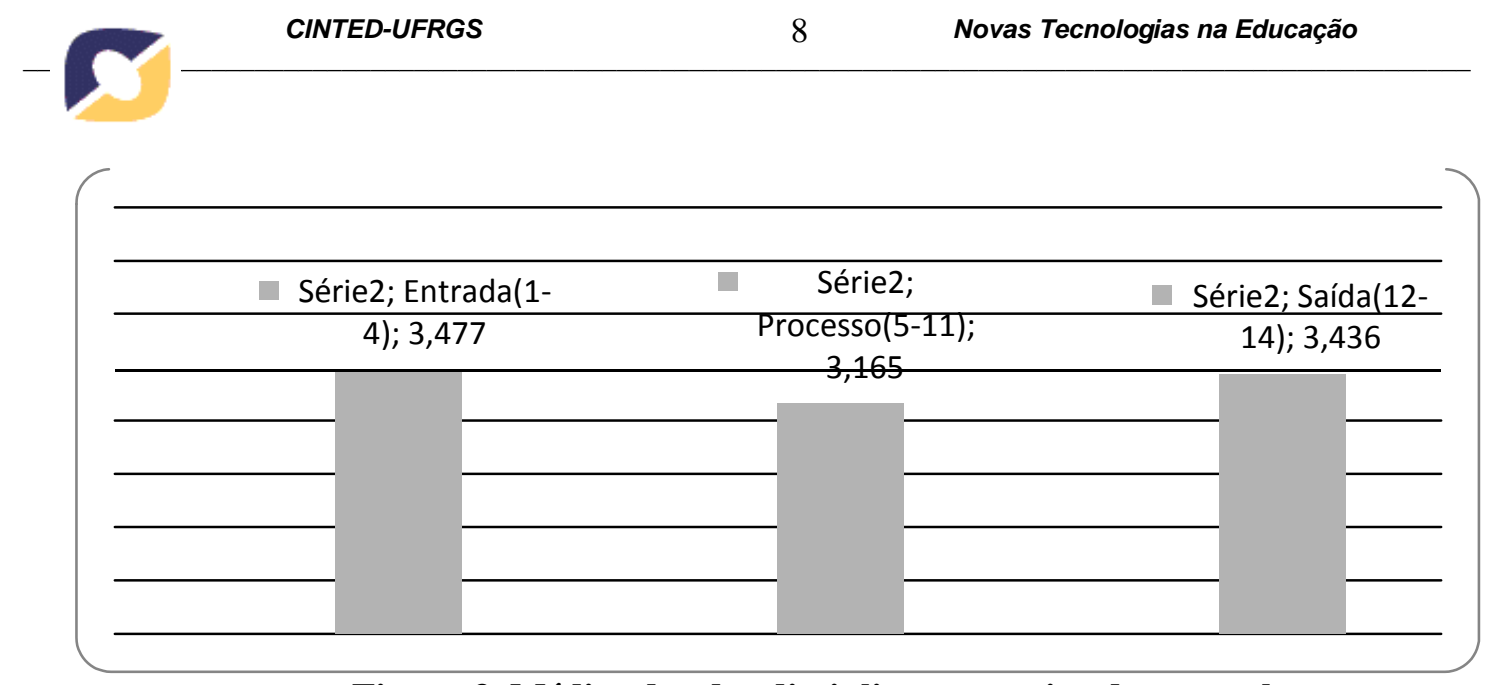

Figura 3. Médias das dez disciplinas com pior desempenho

A similaridade entre as médias obtidas pelos três diferentes conjuntos de disciplinas nos três diferentes tipos de indicadores/aspectos (entradas, processo e resultados), à luz da teoria sistêmica, indicam a validade do instrumento na medida em que o nível da qualidade das entradas e dos processos determinam de forma coerente a qualidade dos resultados obtidos.

\section{Conclusão}

Este artigo apresentou a validação do instrumento de avaliação de disciplinas semipresenciais com vistas a contribuir com o desenvolvimento da avaliação e dos indicadores de qualidade da modalidade a distância. Diferentemente de muitos instrumentos elaborados sem base científica, a validação desenvolvida considerou uma proposta de instrumento e indicadores definidos com base na Teoria Sistêmica onde, de forma geral, entende-se que o nível das "entradas" impacta no desempenho do "processo" que, por sua vez, determina a qualidade dos "resultados".

O próprio reconhecimento da condição privilegiada da semipresencialidade, que permite uma comparação entre as modalidades a distância e presencial, evidencia a adequação do desenvolvimento de estudos sobre instrumentos e indicadores de qualidade com base nessa modalidade. De fato, disciplinas semipresenciais consubstanciam uma condição privilegiada para investigação da qualidade da EaD visto que um mesmo grupo de alunos vivencia, numa mesma disciplina e com um mesmo professor, momentos intercalados entre a modalidade a distância e o ensino presencial.

As médias calculadas para os diferentes conjuntos de disciplinas no contexto dos diferentes tipos de indicadores/aspectos evidenciaram a validade do instrumento proposto como ferramenta de avaliação da qualidade de disciplinas semipresencias. Quando os aspectos de entradas (laboratórios, ambiente virtual, formação do professor etc.) e de processo (didática, interação, acompanhamento, articulação, suporte etc.) são mal avaliados, o instrumento demonstra que a disciplina gera resultados (aprendizagem, desenvolvimento de habilidades etc.) insatisfatórios. Da mesma forma, o instrumento evidencia que uma disciplina com boas entradas e processo gera bons resultados.

Portanto, a validação realizada sobre o instrumento apresentado neste artigo demonstrou a adequação da aplicação da Teoria Sistêmica como base conceitual da avaliação para disciplinas semipresenciais e, por conseguinte, como referencial para o desenvolvimento da avaliação da qualidade da modalidade a distância. 


\section{Referências}

BERTOLIN, J. C. G.; DE MARCHI, A. C. B.Uma Proposta de Indicadores para Avaliar a Qualidade de Disciplinas Semipresenciais em Cursos de Graduação. Revista Brasileira de Computação Aplicada, v. 1, p. 30-41, 2009.

BERTOLIN, J. C. G.; DE MARCHI, A. C. B. Instrumentos para avaliar disciplinas da modalidade semipresencial: uma proposta baseada em sistemas de indicadores. Avaliação (UNICAMP), v. 15, p. 1-10, 2010.

BRASIL. MEC. PORTARIA No 4.059, DE 10 DE DEZEMBRO DE 2004.

BRASIL.INEP. Na Medida - Boletim de Estudos Educacionais do Inep. Disponível em: <http://www.inep.gov.br/download/na_medida/BNMedida-ano1-N2-1Jul2009.pdf> Acesso em: 10 mar. 2010.

ESTRADA, L. R. G. Hacia un modelo de evaluación de la calidad de instituciones de educación superior. Revista Iberoamericana de Educación - Número 21, Universidad siglo XXI, OEI Ediciones, Dez. 1999.

EUROPEAN COMMISSION. European Report on Quality of School Education. Sixteen quality indicators. Luxembourg: European Communities, 2001.

GARCÍA, M. G. Evaluación y Calidad de los Sistemas Educativos. In: RAMÍREZ, Teresa G. (Org.). Evaluación y gestión de la calidad educativa. Málaga: Ediciones Aljibe, 2000.

MIZIKACI, F. A systems approach to program evaluation model for quality in higher education. Quality Assurance Education, vol. 14, no. 1., p. 37-53., 2006.

NAVARRA. Sistema de indicadores de la educación de Navarra 2003. Navarra: Gobierno de Navarra, 2004.

ORGANISATION ECONOMIC CO-OPERATION AND DEVELOPMENT (OECD). Education at a glance: OECD indicators 2002. Paris, 2002.

UNITED NATIONS EDUCATIONAL, SCIENTIFIC AND CULTURAL ORGANIZATION (UNESCO). Marco conceptual. Documentos - Laboratorio Latinoamericano de Evaluación de la calidad de la educación. Santiago, Chile: Lecce, Orealc/Unesco, 1997.

VALCHEVA, D. and TODOROVA, M. Defining a system of indicators for evaluation the effectiveness of e-learning. CompSysTech, 2005. Disponível em: $<$ http://ecet.ecs.ru.acad.bg/cst05/Docs/cp/sV/V.14.pdf >. Acesso em: 11 mar. 2010. 